\title{
Platelet Hyperaggregation and Morphological Changes by Electron Microscopy in Simulated Dive
}

\author{
García de Paoletti Diana $\mathrm{N}^{1 *}$, Espinosa $\mathrm{C}^{2}$, Mauvecin $\mathrm{G}^{3}$, Paoletti \\ $\mathrm{E}^{4}$, Lagens $\mathrm{R}^{5}$, Cabeza Mercket $\mathrm{P}^{6}$, Garcia $\mathrm{CW}^{7}$, Brandon $\mathrm{J}^{8}$ and \\ Suarez Gonzalez Lissbett ${ }^{9}$ \\ ${ }^{1}$ May 25 Laboratory-CEDEAC, National University of Mar del Plata, Argentina \\ ${ }^{2}$ Department of Biochemistry, Director CMH Medicina Hiperbárica SRL, Argentina \\ ${ }^{3}$ Physician Medical Director CMH Medicina Hiperbárica SRL, Argentina \\ ${ }^{4}$ Medical Hematologist, Dean Emeritus University FASTA, Argentina \\ ${ }^{5}$ Physician, President of the Favaloro Foundation, Buenos Aires, Argentina \\ ${ }^{6}$ Department of Biochemistry, Favaloro Foundation, Argentina \\ 7Physician, Director of the Regional Blood Center, Argentina \\ ${ }^{8}$ Department of Biochemistry, Submarine and Diving School \\ ${ }^{9}$ Medical Clinical Haematologist, Argentina
}

*Corresponding author: García de Paoletti Diana N, Department of Biochemistry, National University of Mar del Plata, Laboratorio de Clínica 25 de Mayo, Mar del Plata City, República Argentina, E-mail: dianangarcia@hotmail.com

\section{Abstract}

Dysbaric osteonecrosis and electroencephalogram (EEG) disturbances prompted the previous studies already submitted at Argentine Society of Haematology on the Scientific Meeting at 1982 and on the First Haemotherapy and ImmunoHaematology Argentine Congress at Buenos Aires in 1986. We will describe the study of platelet aggregation and platelet morphological changes by electron microscopy before and after of simulated dive in hyperbaric chamber without the addition of aspirin (ASA).

Materials and Methods: One hundred thirty (130) divers were studied at the Argentina Navy Submarine and Diving School (AA), in groups of ten (10) during the training courses required by the AA. Air was used as the breathing gas and all the dives were at a max depth of 39 meters (4.9 ATA-Absolute atmospheres) with a decompression was made according to US Navy Decompression Tables in use in the AA. First decompression stop at 6 meters (1.6 ATA) for 10 minutes and the second stop at 3 meters (1.3 ATA) for 25 minutes. Partial pressure of oxygen (Pp $\mathrm{O}_{2}$ ) remained constant at the bottom to 1.03 ATA and partial pressure of carbon dioxide (Pp CO2) below 0,025 ATA. Blood was obtained in sodium citrate $\mathrm{Na} 3.2 \%$ at a rate of $1: 9$ citrate / blood. 
Conclusion: We found that the simulated dive in vivo produces hyper aggregation and morphological changes in platelet in divers who did not take ASA.

Keywords: Platelet Hyperaggregation; Haemotherapy; Immuno-Haematology; Electron Microscopy

Abbreviations: EEG: Electroencephalogram; ASA: Addition of Aspirin.

\section{Introduction}

\section{Platelet Morphology}

These are cellular fractions originated in megakaryocytes, usually have an ovoid shape, with a diameter of about 2-4 microns; they do not have a nucleus and the rough endoplasmic reticulum is very scarce, morphologically they present a granular appearance to the optical microscope, but with electronic microscopy it is possible to observe a delimiting membrane and an organelle content. Said membrane constituted by three sheets of a total thickness of 100 to 200 Armstrong; internally, a circular band of microtubules serves as a structural scaffold, which helps maintain its ovoid shape. In the periphery to the membrane exists a coating of about 500 Armstrong of thickness, of plasmatic origin, that forms a species of mantle or intimately around atmosphere that is constituted by mucopolysaccharides or glucose amino acids and glycoproteins that suspects could intervene in the processes of platelet aggregation. From the functional point of view, the platelet membrane, apart from the maintenance functions of the platelet shape, also has the property of reacting selectively to external stimuli, since they are equipped with specific receptors for ADP, adrenaline, 5- hydroxytryptamine, Fc fragment of immunoglobulins and at the same time being the seat of important enzymatic activities involving adenyl cyclase, phosphodiesterase, ATP, ASA, acid phosphatase, phospholipase A2, glucosyl transferase, etc. In addition, fibrinogen, coagulation factor VIII, platelet actomyosin, arachidonic acid, which is intimately linked to the metabolism of prostaglandins and has components of the cAMP system, for the transport of $\mathrm{Ca}++$ linked to the moment of platelet aggregation and finally a thrombinsensitive glycoprotein.

Below the platelet membrane there is a system of open vesicles or membranous channels, connected to each other and to the surface, a channel that is called a surface connection system, one of whose functions would be to considerably increase the contact area necessary for the exchange. In relation to this system there is a second one, which probably derives from the endoplasmic reticulum, which is called dense tubular, which is attributed to be involved on regulating the intracytoplasmic calcium levels and on whose walls the prostaglandin and thromboxane were located. In the platelet cytoplasm, different organelles can be distinguished, such as mitochondria, alpha granules, dense bodies, glycogen granules and the two systems previously mentioned. Experimental separation of the different types of granules is carried out by carefully breaking the outer membrane of the platelets with appropriate means, collecting the intracytoplasmic organelles and subjecting the material thus obtained to centrifugation in a medium of sucrose in a density gradient. Granules shall be located where there is a coincidence of density between the granule and the sucrose concentration. Granules that have shown the highest density in electron microscopy are the densest gradients; their content is given by a mixture of serotonin, calcium, ADP, ATP, etc. But it is interesting to note that these two nucleotides (ADP and ATP) do not exchange with the corresponding ones in the cytoplasm. These granules also contain serotonin, which is synthesized by the cells of the intestinal wall, circulates through the blood and is captured by the outer membrane of platelets; it is also not exchanged with the neurotransmitter serotonin in the brain.

Other intracytoplasmic granules, which are called alpha, are lighter and more numerous than dense ones, they contain different proteins, probably synthesized in megakaryocytes; some of them are only found in platelets, as is the case with platelet factor 4, which counteracts heparin. Another proper factor is found in the granules: it is b thromboglobuline and other proteins similar or dentical to plasma proteins that are involved in the coagulation process, such as fibrinogen. A third group of granules is given by lysosomes, which, as in other cells, contain enzymes that hydrolyze proteins and complex carbohydrates.

The platelet form is maintained by a cytoskeleton, whose microtubules are arranged in bundles and sometimes in spiral form, with bridges connecting them. Each microtubule consists of about 10-15 filaments. Inside are the microfilaments, related to actomyosin, 
whose functions are the contraction of platelets, a fact that becomes evident when they meet or agglutinate.

Platelets contain a protein, factor 4 , which neutralizes heparin, and is associated with a membrane transporter, which has not been found in dense groups, but can be found in granules and plasma after platelet aggregation. Actomyosin is a very important contractile protein in platelet physiology, making up about 15 to 20 percent of total platelet proteins. Many activities are carried out, such as pseudopod formation, aggregation, clot retraction, etc. Another important element is calcium, currently estimated that the contractile and secretory processes are mediated by an increase in the concentration of cytoplasmic calcium, which is provided from the deposit sites in the sarcoplasmic reticulum, mitochondria, etc. The role of calcium in platelet function is complex and therefore, the first stage of platelet aggregation does not occur in the absence of calcium, as opposed to the release reaction that can occur even in the absence of calcium.

\section{Platelet Physiology}

The functions of platelets are performed in close relationship with the normal state of the endothelial wall of blood vessels, whose alteration may be the result of the aggression of the most varied noxas, among which we can mention the carbon monoxide component of cigarette smoke, lipids, circulating fatty acids, etc. These noxas ultimately expose the subendothelial layers; when the continuity solution is produced on the surface, elements of the subendothelial conjunctiva will be discovered, whose collagen interacts with the platelets, these platelets quickly adhere to it and to other platelets that cross the area of aggression, forming the hemostatic plug in approximately one minute, under normal conditions.

The adhesion of the platelets to the damaged vascular wall is an enzymatic process whose initiation is determined by the reaction of galactosic groups terminating the lateral collagen chains forming a complex, of the reactions of the hemostatic process. Factor VIII also acts as a coadjuvant at the beginning of this process, after modifying its molecule.

The pathophysiological sequence of this platelet process is as follows:

a) Change in the shape of platelets;

b) Adhesion to subendothelial components;

c) Primary platelet aggregation;

d) Reaction of release of ATP, ADP and amines Phase I

e) Secondary or irreversible aggregation Phase II
Changing the shape of the platelets: from the discoid shape, more or less regular, they become spheroidal elements, which are softened at first, and later on they will emit cytoplasmic extensions, like pseudopods, to finally give them the appearance of a thorny sphere. Fibrinogen-specific receptors appear on the surface. At this stage, they can adhere to each other or to foreign surfaces, reaching the stage of platelet aggregation. These shape changes are induced by ADP, thrombin, 5hydroxytryptamine, collagen components and prostaglandins. The presence of specific receptors for fibrinogen is demonstrated by the binding capacity shown by platelets that have been stimulated by ADP and fibrinogen, which at this time would act as a kind of binding cement between platelets. The fibrinogen molecule is capable of such a function because it is a dimer, a double molecule, each of which could bind with other molecules, encompassing platelets and red blood cells. When the transformation of fibrinogen into fibrin occurs by the action of thrombin and its subsequent polymerization, it is first achieved by the release of fibrinopeptides (A and B) from fibrinogen, thus giving a monomer of fibrin; the exposed chain regions are used for bonding with other fibrin monomers thus forming the first fibrin dimer of fibrin, then the polymers and finally the fibrin strands. Adhesion to subendothelial components: such as myocells, conjunctive fibers, basement membrane, and others. For this property of adhesion to manifested, the presence of cofactors such as calcium, fibrinogen, factor VIII of coagulation and von Willebrand factor is necessary, to which it is associated.

\section{Primary Platelet Aggregation}

It can occur in two stages or phases. One moderate that occurs after 10 seconds and the other more intense that takes place the minute after. The first is induced by thrombin, adrenaline, ADP, 5-hydroxytryptamine, collagen, immunocomplexes, etc. and it requires the presence of calcium and fibrinogen. This stage has its inhibitors including adenosine, cAMP and some prostaglandins.

ATP, ADP and amine release reaction: This conditions a second phase of this platelet aggregation process, which is characterized as irreversible. In this process, about 30 seconds the platelets approach, but while retaining their own individuality, the intracytoplasmic organelles move towards the center of the platelet. Later begins a period of irreversible aggregation in which the platelets lose their characteristics, merging with each other by means of the pseudopods that intertwine, ending up forming accumulations with the impossibility of recognizing their individuality. 
a) Phase I can be blocked by anti-inflammatory drugs such as acetylsalicylic acid, dipyridamole, sulfinpyrazone and ticlopidine.

b) Phase II is induced by calcium, thrombin and collagen; its inhibition being is more difficult due to nonsteroidal anti-inflammatory drugs.

Aggregation and release of platelets are inhibited by cAMP and other analogues. The observation that thrombin and collagen can directly inhibit adenyl cyclase of the membrane and thus potentially decrease for cAMP suggests that platelet aggregation is related to cAMP values, but to date it has not been adequately clarified how the aggregated factors decrease cAMP values. Although the beta/delta receptor activated by the peroxisome proliferator (PPARbeta / delta) is a nuclear receptor found in platelets and the levels of cAMP are specifically mediated by PPARbeta / delta. Diving is a dangerous activity not only due to the difficulties for a terrestrial mammal, such as a human being, found when venturing into an aquatic environment for which he is not adapted but also because there are many pathologies associated to this activity.

Such pathologies can develop during descent or permanence at depths (drowning, intoxication caused by respiratory gases, barotrauma), during ascent as aeroembolism, decompression sickness and a posteriori as dysbaric osteonecrosis $[1,2]$ that can develop in the long term.

Disbaric osteonecrosis is much less common in sport divers than in commercial or military divers and the rate may differ from $2.5 \%$ in the U. S. Navy. Up to $80 \%$ doubtful in Chinese commercial diving [3]. But it is also interesting to note that the SCUBA (Self Contained Underwater Breathing Apparatus) diving has been introduced as a sport, has become very popular and in the United States there are only 17,000,000 sport divers. Necrotic bone lesions in patients with high doses of steroids are not the same as those seen in dysbaric disease 3. The mechanisms involved in the development of a vascular osteonecrosis after hyperbaric exposure have not been clarified; it is true that in decompression illness the coagulation mechanisms are activated and there is a relationship between inadequate decompression and the development of dysbaric necrosis. A well-known phenomenon is that when a diver decompresses, bubbles occur and decompression sickness has always been linked to the uncontrolled ascent of the diver to the surface.

There are theories to explain this pathology. Air embolism during decompression, fat embolism caused by the bubbles within the bone marrow and the occlusion of intraosseus capillaries; the silent intravascular bubbles activate the coagulation cascade in the following sequence:

a) damage of the endothelial cells caused by the bubbles

b) platelet thrombus and secondary deposition of fibrin

c) microvascular occlusion and

d) Arterial narrowing as a result of intimal damage [4]

Experiments in rats have shown intracerebral platelet aggregation when the rats were subjected to sudden decompression [5]. The effects of different bubbled gases $\left(\mathrm{N}_{2}, \mathrm{He}, \mathrm{Ne}, \mathrm{Ar}\right.$, or a mix of $\left.\mathrm{O}_{2+} \mathrm{CO}_{2+} \mathrm{N}_{2}\right)$, are equally potent platelet agonists varying depending on the inhibitors used or the presence of proteins, mainly fibrinogen. Platelet activation has been proposed to play a significant role in the pathogenesis of thrombotic conditions and is currently recognized as the probable origin of the Divers Decompression Sickness [6].

Being aware of the existence of dysbaric osteonecrosis and after finding electro- encephalographic changes is some experienced divers of the Argentine Navy we decided to embark on the study of platelet function in 1982. Our first studies showed increased platelet aggregation after a simulated diving run [7-10].

Simulated diving poses little risk of physical trauma and is a mandatory practice as part of the Argentine Navy training program under controlled risks therefore no decompression accidents or trauma were seen in any of the divers studied. Morphological changes on platelets aspect, were observed by Electron Microscopy (EM) Thorsen, et al. [11], similar to those produced by the classical agonist such ADP, collagen and thrombin.

\section{Materials and Methods}

The study included 130 divers of the Submarine and Diving School of the Argentine Navy divided into groups of 8 to 10 subjects each group. All of them volunteers. They were studied during the hyperbaric chamber tests (part of their usual training) by drawing blood samples before and after the simulated dive.

The same diving profile was used in every single case with the same respiratory gases (air) and pressure: 4.9 ATA (39meters) for 39 minutes bottom time. The Decompression phase was performed according to the EDU Tables of the USA Navy (USNAVY DIVING MAUAL Rev 5 2005) that was uses in the navy. The PPO 2 remained constant at 1.03 


\section{ATA}

PPCO2 remained below 0.025 ATA by means of a regeneration system and an infrared absorption $\mathrm{CO} 2$ analyzer Shlumberger Anir 12. The blood was obtained by puncture of the ulnar vein in sodium citrate at $3.2 \%$ in a ratio of citrate / blood: $1 / 9$ and analyzed in a Chronological Aggregation meter. For MS, PRP (plateletrich plasma) was obtained from centrifugation samples, 30 minutes to $1000 \mathrm{~g}$. The sediment was fixed in glutaraldehyde at $2.5 \%$ in phosphate buffer $0.1 \mathrm{M}, \mathrm{pH} 7.2$, was subsequently fixed in $1 \%$ osmium tetroxide in the same buffer and was included in epoxy resin to obtain ultrafine cuts which were dyed with lead nitrate and studied under electron transmission microscope. The following laboratory determinations were made:

a) Platelet count [12]

b) Prothrombin time [13]

c) Partial thromboplastin with kaolin partial time (APTT) [14]

d) Spontaneous platelet aggregation [15]

ADP-induced platelet aggregation (2x10-4mole/litre) without and with previous intake of $600 \mathrm{mg}$ acetylsalicylic acid (AAS). Epinephrine-induced platelet aggregation (1x10-4moles/litre) without and with previous intake of $600 \mathrm{mg}$ acetylsalicylic acid (AAS). ADP-induced platelet aggregation (2x10-4moles/litres) without and with previous intake of $325 \mathrm{mg}$ acetylsalicylic acid (AAS).

a) Platelet microaggregates [16]

b) Antithrombin III [17]

c) Serum lactic dehydrogenase (SDL).

d) Free plasma hemoglobin [18]

e) Platelet aggregation induced by ADP (2x104 moles/litr) without and with the previous intake of $250 \mathrm{mg}$ of ticlopidine hydrochloride.

f) Platelet aggregation induced by ADP (2x10-4 moles/litre) without and with previous intake of 130 mg of sublingual ASA.

g) Electron microscopy of platelets before and after simulated hyperbaric chamber dives

\section{Aggregation}

\section{Cyclo-oxygenase Exists in Isoforms}

Cyclo-oxygenase 1 (COX-1), constitutive, mainly in platelets and cyclo-oxygenase 2 (COX-2), inducible in several cell types, including endothelial cells. ASA is rapidly absorbed in the stomach and small intestine by passive diffusion through the gastrointestinal membranes. AAS has a short half life (15-20 minutes) in circulation, but its inhibitory action on COX-1 is 50 to 100 times more potent than on COX-2. Therefore, it is an ideal drug to act on the anucleated platelets, decreasing the formation of TXA2. In addition, ASA probably inactivates COX-1 in mature megakaryocytes and as the platelet pool renews approximately $10 \%$ daily, a single daily dose of ASA is able to maintain the inhibition of TXA2 formation. Therefore, despite the short half-life in circulation, its antiplatelet action is greater. Thus, there is dissociation between the pharmacokinetics and pharmacodynamics of ASA. The patient who takes AAS may have the following results from the tests on platelet aggregation.

Platelet aggregation: the response is dose-dependent, as is also a function of the underlying pathology by which the patient is antiaggregated.

\section{In general it is}

Adrenaline (ADR): 1 Normal wave, 2 wave Absented

ADP: 1 Normal wave, 2 wave Disaggregate or irreversible response It is controversial the fact that resistance to Aspirin can be observed, the actual biochemical resistance measuring the activity of platelet COX-1 has been observed in a very low percentage, although it has been seen in some series between 5-60\% of the patients who had an insufficient response measured by aggregation, usually atherosclerotic patients, who generate a very strong platelet activation, which is not our case, which are normal, healthy and studied clinically and under cardiac point of view.

Determinations were made immediately before and after the dive (Tables $1 \& 2$ ).

\begin{tabular}{|c|c|c|c|c|}
\hline \multicolumn{2}{|c|}{ Without Aspirin } & $\%$ total & \multicolumn{2}{c|}{ With Aspirin } \\
\hline ADP & Epinephrine & & ADP & Epinephrine \\
\hline Out of range & Out of range & $68 \%$ & $100 \%$ & $30 \%$ \\
\hline $120 \%$ & $130 \%$ & $14 \%$ & $80 \%$ & $20 \%$ \\
\hline $80 \%$ & $90 \%$ & $18 \%$ & $50 \%$ & $4 \%$ \\
\hline
\end{tabular}

Table 1: Platelet's aggregation percentage with and without ASA after diving.

\begin{tabular}{|c|c|c|c|c|}
\hline Study & $\begin{array}{c}\text { Before } \\
\text { (average) }\end{array}$ & $\begin{array}{c}\text { After } \\
\text { (average) }\end{array}$ & Student Test p & No \\
\hline TP \% & 88 & 76.4 & 0.389 & 40 \\
\hline APTT & 42.5 & 45.2 & 0.081 & 40 \\
\hline PLATELETS. & 275 & 320 & 176 & 40 \\
\hline
\end{tabular}

$\mathrm{TP}=$ Prothrombin time, APTT (activated thromboplastin time) and platelet counts.

Table 2: There were no changes in global coagulation tests, after immersions. 
The antithrombin III, LDH, free plasma $\mathrm{Hb}$ and spontaneous platelet aggregation showed no significant differences in samples taken before and after diving with or without aspirin intake not represented. A group of divers were given $600 \mathrm{mg}$ aspirin (ASA) PO two hours before the dive. The results showed that only $11 \%$ of divers them had alterations in platelet aggregation seen in the rest of the divers, probably due to the action of large doses of ASA in the vascular endothelium. Platelet aggregation was quantified across the area below the curves. The area under the curve was measured at the time of adding the agonist for up to 300 seconds. This area was used to develop the graphics. Saad technique E ET AL 15 (modified).

\section{Electronic microscope}

This report shows that compression-decompression in vivo causes a significant alteration of the platelet morphology with loss of its normal form shape, disappearance of microvilli and the adoption of a concave-convex shape (similar to a sickle) plus the loss of ? ? ?and dense granules and dense. Granular loss can be attributed to platelet activation. The increase in hydrostatic pressure, an infrequent factor among the causes of platelet aggregation, may be involved in the peculiar modification of the form or also the granular loss may be attributed to platelet activation by micro bubbles (Figures 1-6).

Figure 1 shows the results of non-AAS platelet aggregation of the group whose blood samples were studied under the electron microscope.

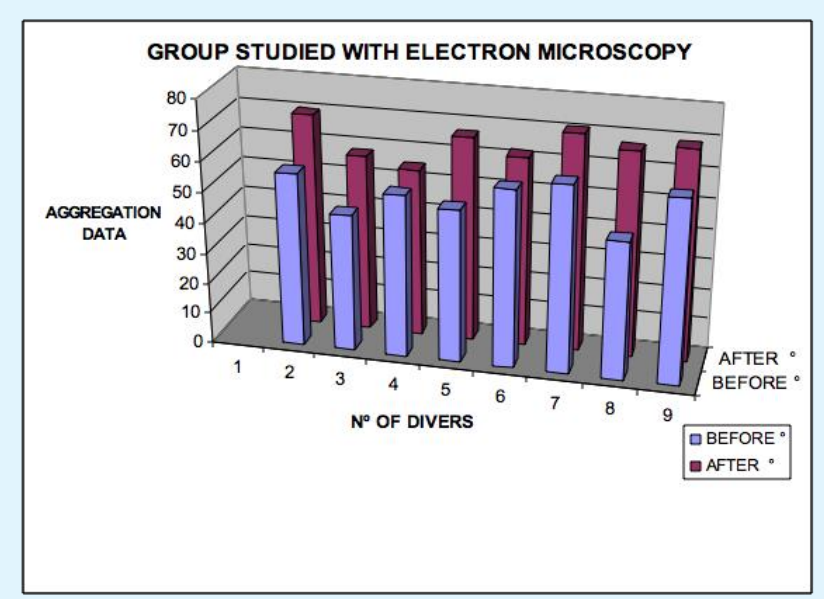

Figure 1: The results of non-AAS platelet aggregation of the group whose blood samples were studied under the electron microscope.

\section{Before Diving}
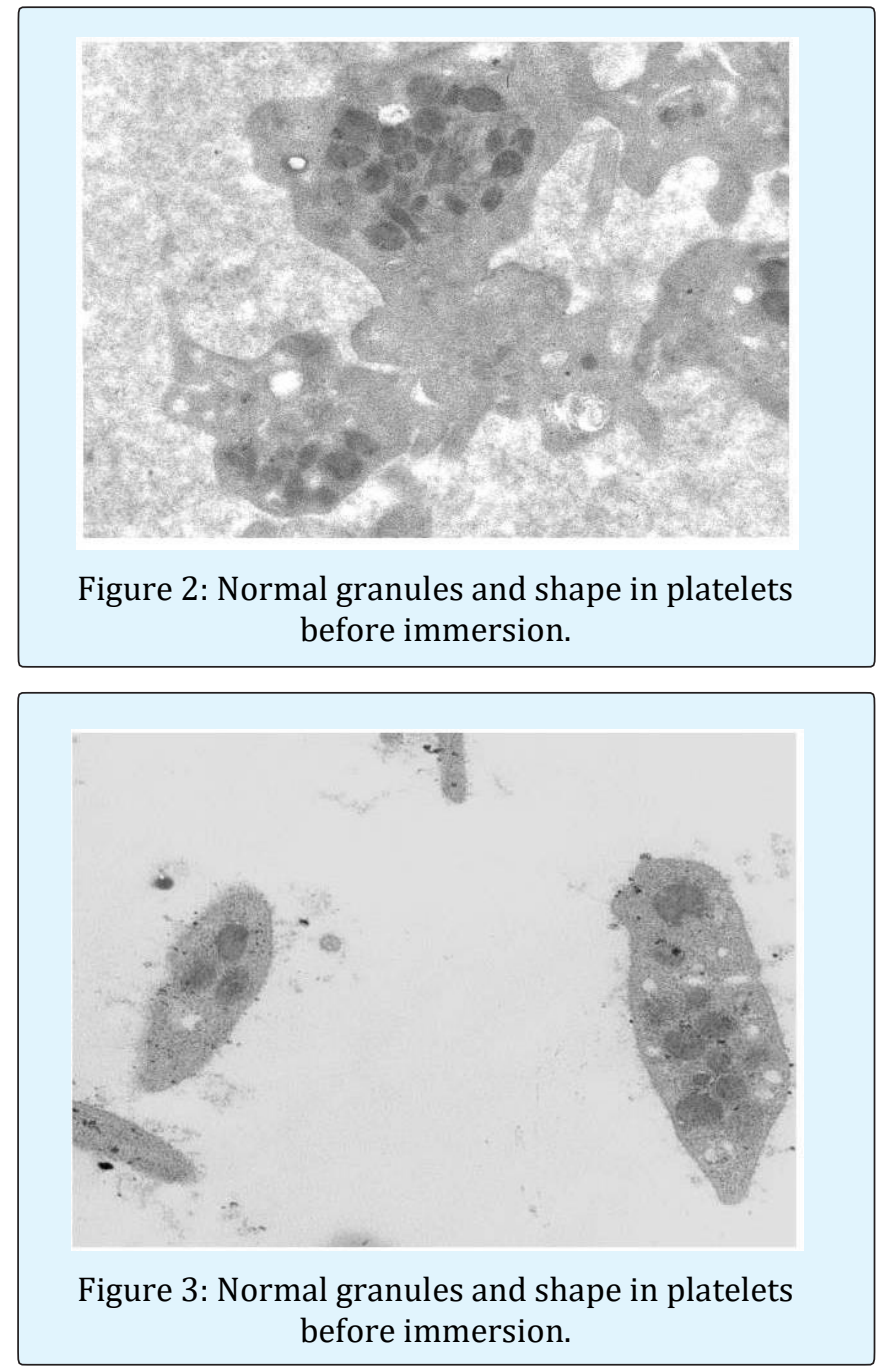

\section{After Diving}

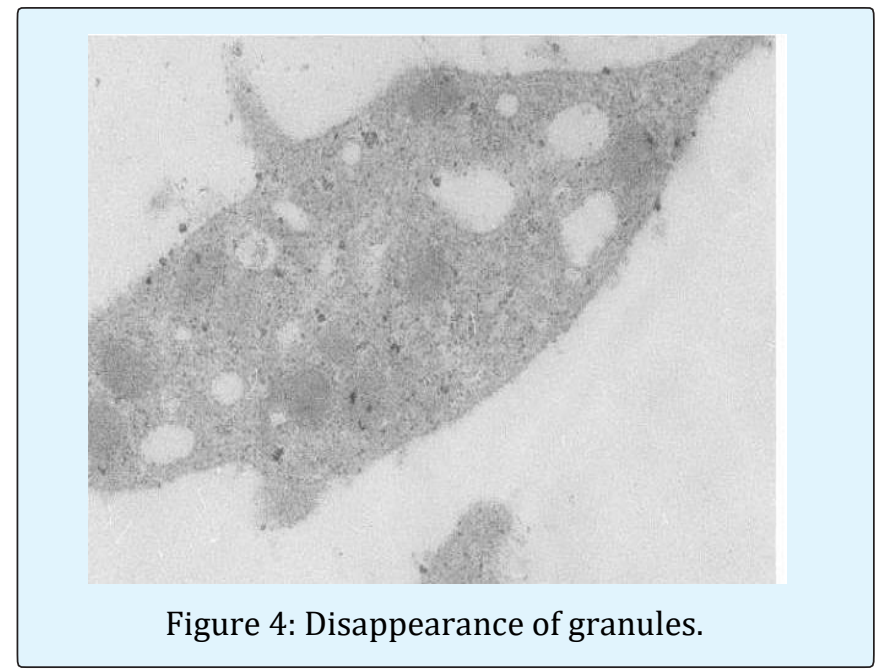



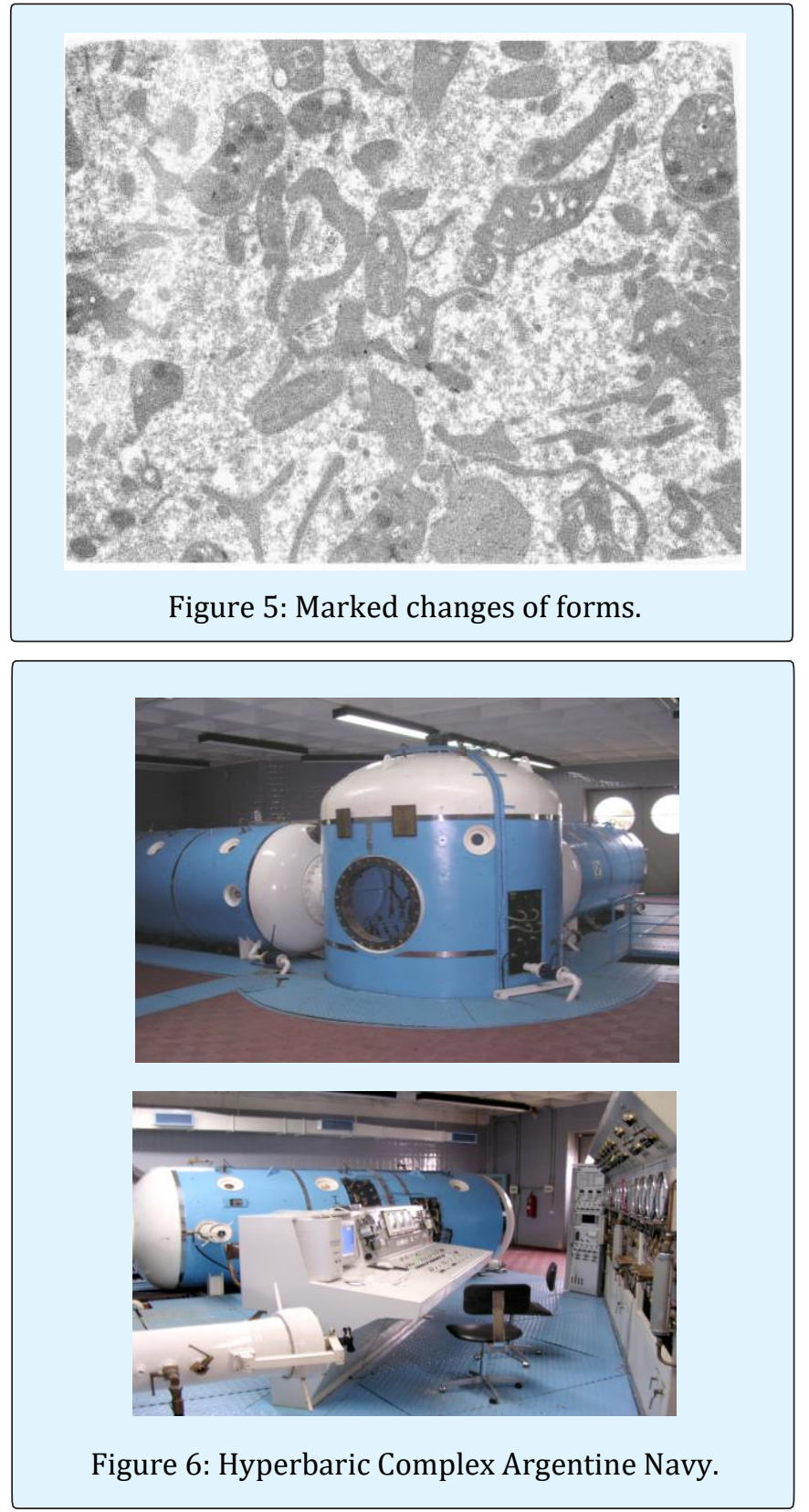

\section{Conclusion}

We found that the simulated dive in vivo produces hyper aggregation and morphological changes in platelet in divers who did not take ASA. The morphological changes by electronic microscopy were deformation of the microvillus, making concave-convex shape (sickle aspect like) and loss of a and dense granules similar to those observed by contact with agonists. The granular loss can be attributed to platelet activation by micro bubbles.

\section{References}

1. Kelly M (1957) George Edward Twynam (18561941), of Sydney: decompression disease of bone. Med J Aust 44(5): 164-165.

2. Edmons C, Lowry C, Pennefather J, Walker R Diving and Subaquatic Medicine $4^{\text {th }}$ (edn), pp: 167-182.

3. Slichter SJ, Stegall P, Smith K, Huang TW, Harper LA (1981) Dysbaric osteonecrosis: a consequence of intravascular bubble formation, endothelial damage, and platelet thrombosis. J Lab Clin Med 98(4): 568590.

4. Bateman J (1951) Review of data on value of preoxigenation in prevention of decompression sicknes, In: Fulton J (Eds.), Caisson sickness, diver's and flier's bends, and related syndromes Philadelphia, Saunders WB pp: 242-247.

5. Thorsen T, Klausen H, Lie RT, Holsem H (1993) Bubble-induced aggregation of platelets; effects of gas species, proteins, and descompression. Undersea Hyperb Med 20(2): 101-119.

6. Bosco G, Yang ZJ, Savini F, Nubile G, Data PG, et al. (2001) Environmental stress on diving-induced platelet activation. Undersea Hyperb Med 28(4): 207-211.

7. Paoletti E, Espinosa C, Garcia D, Garcia W (1982) Estudio de los efectos de la descompresión sobre la Hemostasia. Presentación previa Soc Arg de Hematología Bs As.

8. Paoletti E, Espinosa C, Garcia D, Mauvecin G (1988) Estudio de coagulación en buceos normales. XVIII Jornadas Científicas del Interior. Sociedad Argentina de Sanidad de las Fuerzas Armadas. Mar del Plata.1985

9. Paoletti E, Espinosa C, Garcia D, Garcia W, Mauvecin G (1988) Alteraciones de la función plaquetaria, luego de buceos simulados en cámara Hiperbárica. I Congreso Argentino de Hemoterapia e Inmunohematología Buenos Aires.

10. Paoletti E, Espinosa C, Garcia D, Mauvecin G, Brandon J (1988) Estudio de la agregación plaquetaria luego de buceos normales. En colaboración I Congreso Panamericano de Medicina Hiperbárica y Buceo Buenos Aires. 
11. Thorsen T, Dalen H, Bierkvig R, Holmsen H (1987) Transmission and scanning electron microscopy $\mathrm{N}_{2}$ microbubble-activated human platelets in vitro. Undersea Biomed Res 14(1): 45-58.

12. Brecher C, Cronkite EP (1958) Morphology and enumeration of human blood platelets. J App Physiol 3(6): 365-377.

13. Quick AJ (1966) Hemorrhagic Diseases and Thrombosis. $2^{\text {nd }}$ (Edn.), LEA and FEBIGER, Philadelphia.

14. Proctor RR, Rapaport SL (1961) The partial thromboplastine time with Kaolin, a simple screening test for first stage plasma clotting factor deficiencies. Am J Clin Pathol 36: 212-219.
15. Miale JB (1977) Laboratory medicine hematology. The Mosby Company St. Louis. Missouri $5^{\text {th }}$ (edn).

16. Philp RB (1973) Bloodbubble Interaction in Decompression Sickness. DIEM, 516, Toronto.

17. Wu KK, Hoak JC (1974) A new method for the quantitative detection of platelet aggregation in patients with arterial insufficiency. Lancet 2(7886): 924-926.

18. Bing FC, Baker RW (1931) Determination of $\mathrm{Hb}$ in minute amounts of blood by Wu method. J Biol Chem 92: 589-931.

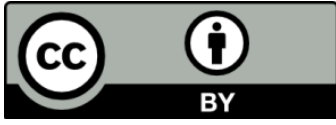

